\title{
Enhancement of Self-organisation in Wireless Networking through a Cross-layer Approach
}

\author{
M. A. Razzaque, Simon Dobson, and Paddy Nixon \\ Systems Research Group \\ School of Computer Science and Informatics \\ UCD Dublin IE \\ abdur.razzaque@ucd.ie, \\ http://www.csi.ucd.ie/users/mohammad-abdur-razzaque
}

\begin{abstract}
Self-organisation has emerged as a very promising approach to the design, deployment, operation, control and evolution of complex wireless networks. The dominant strictly layered design style may however not be able to accommodate many useful optimisations, and we conjecture that cross-layer design may offer a more promising approach. In support of this conjecture we demonstrate through simulation-based approach a cross-layer approach to routing in wireless ad hoc networks that exhibits high degrees of self-configuration, self-optimisation and self-healing. Simulation studies show a substantial improvement in selforganisation properties of wireless networks over comparable layered designs.
\end{abstract}

Key words: Self-organisation, Strict-layer, Cross-layer

\section{Introduction}

The rapid development of new networking technologies is driving towards a world of wireless, mobile, pervasive communications where users can seamlessly and ubiquitously accomplish their tasks, access information or communicate with other users. The current convergence of networked infrastructures and services has changed the conventional view of the network from the simple wired interconnection of a few manually-administered and managed homogeneous nodes to a complex infrastructure encompassing a multitude of different technologies, heterogeneous nodes and diverse services [31]. While users are benefiting from these emerging technologies, the complexity inherent in today's networks creates significant challenges for their design, control, and operations. Due to the resulting increase in the complexity and potential risks associated with the operation and management of such systems, it is becoming increasingly important that these networks have certain self-organisation properties- ranging from selfconfiguration during the startup, to self-adaptation to changes in the operating environment, to self-healing in the presence of component failures or losses- that will minimise the need for human intervention. Such design objectives are fundamental, for example, to the deployment of wireless networks (e.g. Mobile Ad-hoc 
Networks) whose operations are based on ad hoc discovery and routing between network nodes. Self-organisation is also an increasingly important feature for the wired Internet, particularly in the context of peer-to-peer and other emerging applications.

Layering is the key design methodology in communications protocol stacks, but this strict layering is being threatened by next-generation wireless-dominated networking. To obtain self-organisation behaviours in networking systems within existing strictly-layered approaches may be possible, but will not leverage all the possible optimisations. We conjecture that cross-layer approaches with their architectures are better than the existing strict layering approaches in achieving the self-organisation like "self-*" properties in networking systems. This is why the key objective of this work is to show the potential of the cross-layer approaches in enhancing the self-organisation in wireless networking.

Mobile Ad-hoc Networks (MANETs) is one of the key wireless networking environments where self-organisation is very crucial. Interestingly none of the "self*" behviours in networking systems are extremely orthogonal, which means there is some dependency between them. This is why a self-organising system directly or indirectly comprises self-healing, self-configuration even the self-opitmisation $[19,10]$. Moreover, discovery and routing are the two main procedures in selforganisation. So the enhancements to these properties will ultimately enhance the self-organisation in wireless networking systems. Therefore, for the demonstration we will exploit a cross-layer approach to enhance these properties in MANETs.

Cross-layer design breaks away from traditional network design, where each layer of the protocol stack operates independently [32]. In the cross-layer approach information is exchanged between different layers of the protocol stack, and end-to-end performance is optimised by adapting each layer against this information. Cross-layering is not the simple replacement of a layered architecture, nor the simple combination of layered functionality: instead it breaks the boundaries between information abstractions to improve end-to-end transportation. The rest of the paper is organised as follows. Section 2 briefly presents the self-organisation in wireless networking and a summary on self-organising algorithms. In Section 3, we provide a brief description of the cross-layer approach. This section also discusses the possibilities of the cross-layer approach in selforganisation property of wireless networking systems. Section 4 briefly presents some related work. In Section 5, through simulation-based approach we show that cross-layer has the potential to enhance the self-organisation property in MANETs like wireless networking environment. Finally, we conclude and give some directions for future work.

\section{Self-organisation}

Self-organisation can be defined as a process in which the internal level of organisation of a system increases automatically without being guided or managed by an outside source $[18,17,24]$. A self-organising system is organised without any 
external or central control and able to perform complex tasks at the collective level with relatively simple individual behaviours, local interactions without central control or hierarchy. The most significant features of self-organising systems are that there is no central control on the global behaviour of the system; and the global structure and behaviours emerge from local interactions between the different entities or agents that form the whole system without explicit representation of these global patterns at the individual level. Self-organising systems have advantages over conventional systems in terms of robustness, flexibility, autonomy, and the spontaneous development of complex adaptation.

\subsection{Self-organising Networking Systems}

A self-organising network is one that works in an open dynamic environment with no pre-configured support for inter-networking or service location. Different from common self-organising systems, self-organising networks are highly dynamic and distributed, unpredictable, multi-hop and peer-to-peer networks with no central administration and pre-established infrastructure. Cluster or connected dominating set (CDS), tree, grid, or mesh based organisations are typical self- organised network architectures. In a self-organised network, failures, faults and breakdowns must be worked around, and configuration should be adapted optimally in response to the actual state of the network itself and its environment. This requires the support of self-healing and self-configuration in self-organised network. Self-organising communication networks have a range of advantages, for example, they are cost-effective, robust, fault-tolerant, adaptive, flexible, scalable, self-configuring, self-healing, and self-managing. Infrastructure and application are the two distinct level of self-organisation in a network. One is to self-organise the infrastructure level physical networks, which are what commonly concerned in various communication layers; the other is to self-organise the application level networks, which are logically on top of the communication layers. Infrastructure level self-organising networks are to form networks dynamically either based on wireless links for Internet extension and wireless connections for special purposes [33]. Examples include MANETs, Wireless Mesh Networks (WMNs), Wireless Sensor Networks (WSNs), etc. Application level self-organising networks are to form pervasive communicating and ubiquitous service-oriented distributed computing applications in open dynamic environments [34]. Overlay networks, peer-to-peer networks (P2P), distributed pervasive/ubiquitous applications are some examples of this type.

\subsection{Self-organising Algorithms in Networking Systems}

The creation of an ad hoc network and then making it self-organising requires a proper self-organising algorithm. Cluster-based [7, 22] and role-based or taskbased algorithms $[20,25]$ are popular in self-organising networks. A cluster-based self-organisation algorithm groups network nodes into clusters to efficiently forward packets to destinations. In a role-based or task-based self-organisation algorithms assign routing roles or tasks to nodes by sequentially selecting the best 
matched nodes based on their connectivity and computing capacities. Other selforganising algorithms in self-organising networks include location informationaided self-organisation algorithms, biological inspired algorithms and economically inspired algorithms [33].

Discovery and routing are two main procedures in self-organisation algorithms. Discovery is a very important aspect of making an ad hoc network self organising. Discovery deals with two issues:

- Neighbor Discovery: It is the discovery of the node itself and its neighbors within the network. The initial state in a self-organised network is a collection of nodes that are unaware of each other's presence. Thus the first procedure for the nodes to self-organise or join a network is to discover their neighbours which they can communicate with. The simplest way to find neighbouring nodes or to let neighbouring nodes know their presence is to broadcast Hello" message.

- Service Discovery: The discovery of the services available to the node once it has placed itself in the network. Once a node has discovered the network, the next natural aspect for it to look for the services provided to it by the network it is connected to.

Routing is one of fundamental operations of a network. There are numbers of routing protocols proposed to work with ad hoc networks. Link state and distance vector are the two common approaches to solve the routing problem. In the link state each router needs to have a good picture of the network topology but in self-organising networks generally they do not have that and thus does not prove to be a good approach for self organising networks. Routing in selforganised networks is very much different from that in the networks with fixed infrastructure and has the following objectives: (i) it should scale well in terms of the number of nodes and geographical coverage; (ii) routing should have scalable mechanisms that can cope with the dynamism in the network due to mobility; (iii) nodes need to be highly collaborative and redundant, but above all, can not use complex algorithms or protocols. Routing techniques may be broadly divided into proactive and reactive schemes [4]. Proactive protocols have a low delay as the route is already known, but they consume too much bandwidth to propagate the routing information. Reactive protocols use bandwidth efficiently but have a high delay and thus are not suitable for real time traffic. Ideally the routing protocol for a self organising network should be a combination of both the scheme, like the Zone Routing Protocol (ZRP) [15], Cross-layer Self-routing (CLSR) [28].

\section{Cross-layer Design}

The traditional layered design of network protocols is insufficiently flexible to cope with the dynamics of wireless-dominated next-generation communications. Recent studies [12, 27, 32] show that cross-layer approaches are the most promising way out for dynamic network architectures. Instead of treating a layer as a 
completely independent functional entity, information can be shared amongst layers.

\subsection{What is the Cross-layer Approach?}

The approach (in networking) through which a layer in the networking protocol stack (e.g. TCP/IP) can interact or share information with one or more non-adjacent protocol layer/layers is known as the cross-layer approach. This information can be used to adapt protocol functionality in the presence of changing networking conditions, for decision processes such as route selection and as input for algorithms. Through sharing information, mechanisms of different protocols can be combined such as network layer topology maintenance and physical layer power control for example. It is even possible to create new kinds of adaptive applications such as multimedia applications which are sensitive to changing networking conditions. The ability to share information across layers is the central aspect of cross-layer design. So instead of a simple replacement, cross layering can be seen as an improvement of the layered approach. The bottom-line is to preserve the key characteristics of a layered architecture and in addition to allow for performance improvements and a new form of adaptability.

\subsection{Cross-layering in Self-organisation}

Even with its success, strict layering has been criticised as failing to cope with the dynamics of wireless communication and providing too narrow interpretation of the information that can usefully be made use of at a particular layer of abstraction in a networking system $[12,27,32]$. By reducing the information available to a minimum in the interests of simplicity, it is possible that some opportunities for optimisation are lost. In particular, given the rise of "self-*" behaving communications systems like autonomic communications, we would contend that contextual information of vital use in adapting the behaviour of a network to its use and environment is being neglected, and that this acts as a brake on the creation of self-organising, self-adaptive communication systems. Any approach like cross-layering which supports interactions between non-adjacent layers might be helpful in overcoming these obstacles.

Conventional strict-layer based service discovery mechanisms (e.g. [9, 14, 30]) have limited knowledge of the network topology and assume a mostly static environment with infrequent topology changes. In contrast, frequent topology changes are the norm in MANETs like self-organising networking systems, and good service selection is highly dependent on up-to date knowledge of the network topology. Cross-layer based integrated service-discovery will be a better solution in MANETs $[11,16,35]$. By performing service discovery in the same way as route discovery, nodes can accumulate routing information while performing service discovery and disclose service level information at the routing layer, which will improve the overall performances.

Improvement of routing in MANETs like self-organising networking systems is possible. The most desirable routing protocol is one which offers minimal 
end-to-end packet delays for real time traffic and less control overhead for nonreal-time traffic. This can only be achieved by exploiting link state information (such as link life time) that is generally ignored in conventional ad hoc routing protocols: even the existing hybrid protocols do not utilise link state information, and hence do not offer enormous performance advantage over existing reactive or proactive protocols. Exploitation of cross-layering in wireless routing has demonstrated considerable improvement [28, 29].

The configuration in a self-organising networking systems need to be adapted optimally in response to the actual state of the network itself and its environment. A network's state and its environments are the network level and environmental context to the system. This implies that self-organising networks are inherently context-aware. This context can be used to improve performance, adaptability, user satisfactions, and so forth. The strictly layered approaches allow interaction only between adjacent layers and this restricts the possibility of context-awareness within different layers and user. As cross-layer approaches allows non-adjacent layer interactions, so better context-based adaptations and self-cofiguration in self-organising networking systems are possible. Self-healing is needed in self-organising systems to detect, localise, and repair failures automatically. Due to the limited information availability in strict-layer approaches self-healing may not possible. On the other hand cross-layer design can be very useful in this case [6].

\section{Related Study}

So far we have found no work that explicitly addresses the use of cross-layering in self-organising networks. Some works have addressed the cross-layer approaches in improving the service discovery and routing in MANETs. Exploitation of cross-layering in wireless routing has demonstrated considerable potential. Some authors have exploited cross-layering for routing in wireless networks. In [21] a cross-layer approach has been used to exploit mobility information to enhance the performance of AODV (Ad-hoc On Demand Distnace Vector), but considers only constant transmission power which is not true most of the cases. Cross Layer AODV [29] is an efficient routing protocol crossing the routing and MAC sub-layers. A way to improve data accessibility service for a group of mobile users to access desired data has been presented in [8]. To do that it utilises cross-layer assisted predictive location-based QoS routing protocol as well as the replication services. CLSR (Cross-layer self routing) [28] exploits the information related to mobility, power failure and service together in routing for MANETs. On the other hand authors in $[11,16,35]$ have exploited cross-layer approach to improve the service discovery in MANETs. They showed that application and network layer integrated cross-layer based service discovery improves the overall performances compare to the application layer based service discovery. In [6, 13] efforts are made to utilise cross-layer information for self-optimisation and selfhealing respectively. Most of these works are far from considering cross-layer 
design approach for the enhancement of self-organisation in networking systems which really motivates us to do this work.

\section{Demonstration of the Enhancement}

A MANET is a good example of a self-organising networking system and to demonstrate the enhancement of self-organisation using cross-layering we are considering it. Self-organising properties appear in MANETs through its dynamic topology and the routing protocol adapts the topology to the physically possible communication links to organise the network. Thus the routing protocols are directly or indirectly responsible for the self-organising properties in MANET. Routing is therefore an ideal candidate for demonstrating the advantages of cross-layer design in providing self-organisation. For the demonstration purpose we consider the ADOV [2] and the CLSR [28]. AODV is a widely studied strict-layer based routing protocol in MANET environments and CLSR is one of the cross-layer versions of it which exploits link state information and remaining power through cross-layering. Through cross-layer interactions amongst the concerned layers, CLSR the proactively and intelligently maintain the route. This ultimately helps in healing the network without external assistance and improves the route maintenance and also increases the possibility of successful service delivery in MANETs. Due to the limitation of space we are not providing the details of these protocols, please see [2] and [28] for AODV and CLSR respectively. In the following we will be using these two routing protocols to show the improvements in self-organisation in MANETs.

\subsection{Simulation and Results}

To demonstrate the enhancement in self-organisation with the help of a crosslayer approach we use simulation-based approach. For the proper exploitation of cross-layering we need a cross-layer architecture and for that purpose we are using the architecture presented in [26]. The "knowledge plane" is the key element of that architecture through which cross-layer interactions occurs between different non-adjacent layers. The "Global view" of the knowledge plane is responsible for the network related information and the "Local view" is responsible for the node level different layer's information which might be needed during the cross-layer interactions. For example for the dissemination of power warning information in CLSR we need the "Global view" and for the current battery power we need the "Local view".

For the simulation, we have chosen the ns-2.30 [1]. The simulations using Random Waypoint (RW) Model [5] were run in a $1500 \mathrm{~m}$ by $1500 \mathrm{~m}$ area with 50 nodes under varying conditions of mobility. Error bars in the graphs show $95 \%$ confidence interval of the presented values. We did the simulation to show three different aspects of a self-organising networking system and these are: (i) Route Improvement (ii) Self-configuration (iii) Self-healing 
Route Improvement Route breakage is one of the key performance metrics for routing protocols and in this implementation we will show the route improvement using this metric. A MANET is a kind of mobile wireless network in which every mobile node participates in routing operation. Disconnectivity with peer nodes, induced by mobility, power drains and damage makes frequent route breakage. This ultimately interrupts the self-organisation in MANETs and degrade the network's ability to offer services reliably to its peer nodes. Exploitation of information related to the cause of route breakage in routing through cross-layer approach could reduce the route breakage probability and improve the route performance, hence the self-organisation. With the help of CLSR we could show that through cross-layering route breakage reduction is possible. In CLSR, mobility- induced disconnectivity information is contributed by the cross-layering among the network, MAC/link and physical layers, whereas cross-layering between the physical and network layers contributes remainingpower information (for implementation detail see [28]). Using this information, received signal power measurements and a prediction algorithm CLSR could predict the link life time of a link/route and thus it knows the residual life time of a route and the probable route breakage. Finally using the predicted link life time CLSR proactively maintain the route and reduce route breakage. All these together helps in the improvement of the self-organisation in MANETs and reduce service disruptions.

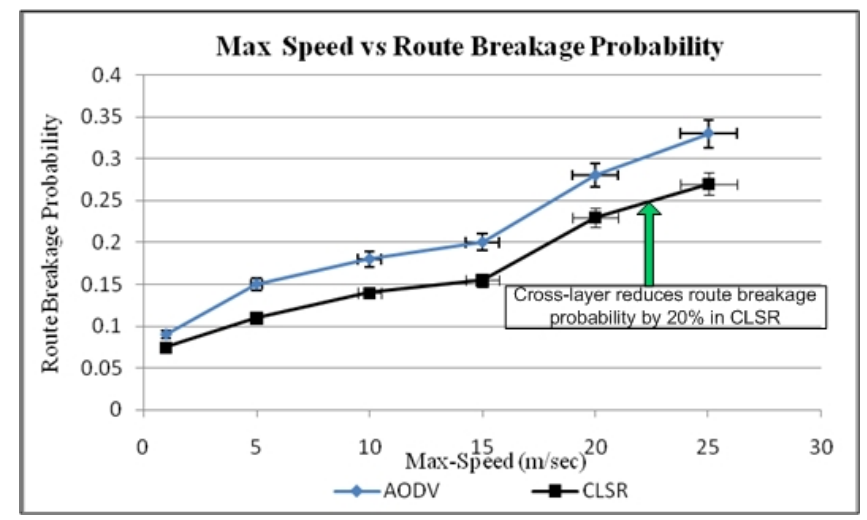

Fig. 1. Route improvement using cross-layering

Figure 1 presents the results for route breakage probability with respect to maximum node velocity/speed. As shown in the graph, for similar network scenario the cross-layer supported routing protocol CLSR shows around $20 \%$ lower route breakage probability than its counterpart strict-layer based routing protocol AODV. AODV deals with the link breakage reactively, whereas CLSR does it proactively, which helps it in advance route discoveries and route repair before the real happening of the link breakage and thus reduces the scope of link/route breakage. The trend in figure shows that route breakage probability increases 
with increasing node speed. This is because with increasing speed, a node moves from its neighbour node's transmission range quickly which increases the route breakage.

Self-configuration Self-configuration is the method for (re-)generating sufficient configurations depending on the current situation in terms of environmental circumstances, e.g. connectivity, quality of service parameters. Self-organisation manifests itself through configuration changes in response to changing user requirements or network conditions. For the adaptation purpose context-awareness is needed. Exploiting network-related context information can make wireless networks like ad hoc simpler, more efficient and more powerful thus simplifying the management of the networking infrastructure for network operators while providing end users with value-added services and an enhanced communication experience. For example, using the networking environments or situational context available resources can be managed according user preferences. In the following we are considering a MANET in a disaster relief operation.

At the times of calamity, rescue and recovery efforts are usually hampered by communications failure as the incumbent communications infrastructure has most likely been damaged or destroyed during the disaster. An ad hoc communications infrastructure, with support for multimedia traffic such as Voice over IP and video streaming, can be placed to support the command, control and communication needs of the rescue and recovery operations. In this paper we are considering a situation where a rescuer is doing VoIP conversion with his mates and also downloading some data (TCP traffic) from his headquarters. VoIP conversation is critical for the rescue effort and it requires minimum QoS support (e.g. minimum bandwidth, delay, etc) and the other application is delay tolerant. During the conversation, for some reasons overall bandwidth of the network drastically falls (due to some obstruction) and if both of the services continue with their existing bandwidth sharing ratio then QoS of the conversation service goes lower than the minimum which will make it useless service. One can solve this problem by closing the downloading application manually but with the help of cross-layer architecture and integration of context-awareness we can self-configure the connections and solve the problem automatically.

Application's bandwidth share management, on a receiving device can be done through the manipulation of the receiver window of its TCP connection such as Receiver Window Control [23]. The receiver reflects its receive buffer status by the advertised window field in the acknowledgments to the sender. If the advertised window decreases, the sender also reduces its send rate. This TCP behaviour can be exploited to reduce the throughput of some applications and consequently increase throughput of rest of the applications, on the receiver. For the above problem we can use this Receiver Window Control. When the link quality goes down then advertising the very small or even zero receiver window for the file download connection will help to increase the bandwidth for the VoIP traffic.

This implementation combines user's (preference) and network's context (dynamic environmental data) and application's QoS requirements to configure the 


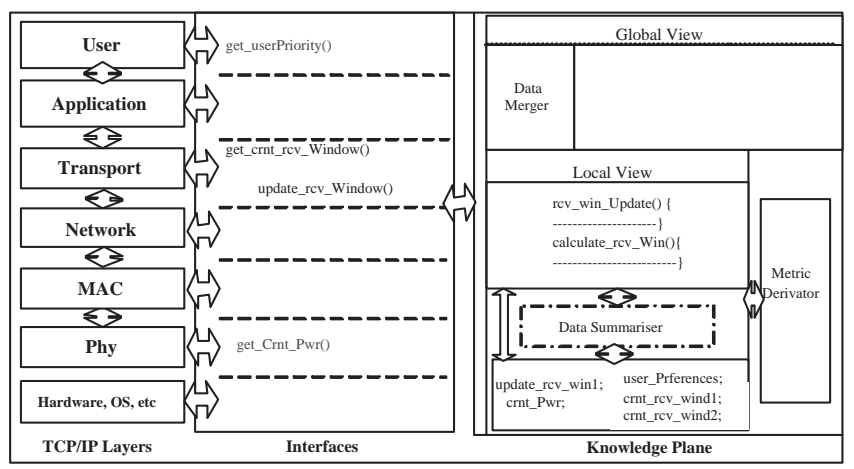

Fig. 2. A snapshot of the implementation of self-configuration using cross-layering

transport layer protocol. To get the network's environmental data, the cross-layer architecture exploits existing link's bandwidth and delay information collected form MAC/Link layer and transport layer. This bandwidth and delay information indirectly presents the environment. In self-configuring the transport protocol in real-time, cross-layer architecture maximises the opportunity that QoS requirements will be met, given the current network situation. During context learning (collection/gathering), the cross-layer architecture considers application QoS levels and the ability of the network to fulfil these requirements. Concern layer related metrics or several management information bases (MIBs) can be used in this purpose. Related application metrics and user preferences populate the "Knowledge Plane" once at the beginning of a communication. Application metrics contains information on the required transmission characteristics (i.e. real-time/non real-time and synchronous/asynchronous), and acceptable worstcase performance metrics (i.e. maximum acceptable bit error rate and maximum acceptable latency), whereas user preferences contains information regarding priority levels of services.

Stepwise interactions for this implementation shown in figure 2 are: (1) Knowledge plane periodically checks the link's bandwidth collected from MAC Layer. (2) If the link bandwidth is lower than a critical value then it collects the end to end delay for the concerned link. In this step, knowledge plane calculates the minimum bandwidth requirements (for the current situation) for the acceptable worst-case performance metrics (i.e. delay jitter for VoIP traffic) and if the total available bandwidth is less than the minimum requirement then knowledge plane does nothing. Otherwise, knowledge plane calculates the additional bandwidth requirements (minimum required bandwidth - existing bandwidth) for the VoIP and on the basis of the priority levels (higher priority for VoIP traffic) collected from the user it also finds the bandwidth share ratio of the two traffic flows. (3) Knowledge plane gets the current receiver window size for the downloading connection from the Transport layer (TCP). (4) Finally, knowledge plane calculates the new receiver window size (reduced) for the TCP connection and updates the Transport layer accordingly. This reduced receiver window 
size ultimately contributes in increasing the bandwidth of VoIP traffic, hence maintain the minimum QoS for the higher priority service (VoIP).

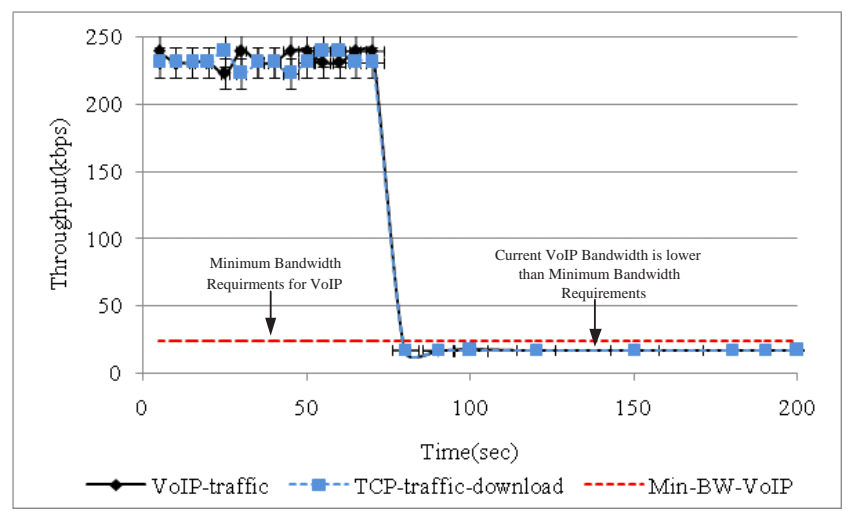

Fig. 3. The problem without cross-layer design

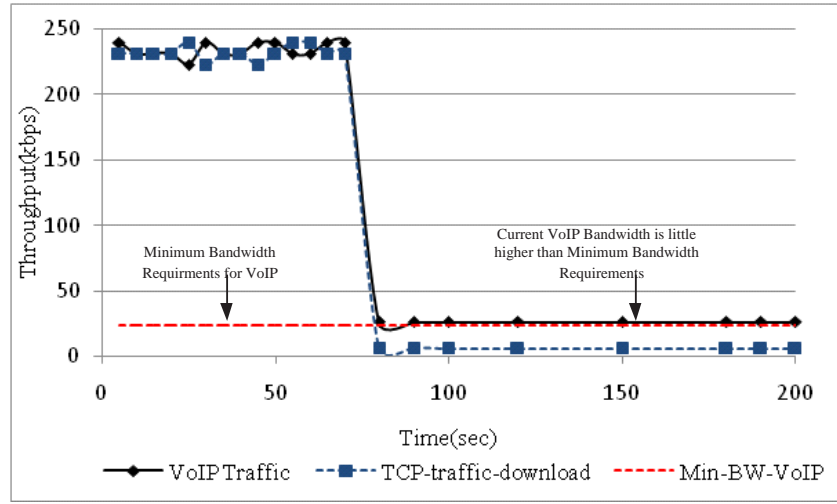

Fig. 4. Result of cross-layer supported self-configuration

Figure 3 shows the problem when there is no cross-layer and both the applications get the almost same bandwidth when the bandwidth falls. As shown in the figure the bandwidth $(16 \mathrm{~kb})$ becomes less than the minimum requirement $(24 \mathrm{~kb}$ [3]). In this situation VoIP traffic, the mission-critical task could not support QoS. Figure 4 is the solution to this problem using the cross-layer implementation. When the link quality as well the link bandwidth goes down, instead of equal sharing of the current bandwidth between the applications, it utilises QoS requirements and user context (priority) to self-configure and distribute the available bandwidth. After the redistribution of bandwidth VoIP traffic gets around $26.1 \mathrm{~kb}$ whereas TCP traffic gets around $6.2 \mathrm{~kb}$, which means VoIP can 
maintain the minimum QoS. Being a real-time traffic VoIP flow can not tolerate delay but the other one can. Therefore, real time traffic gets the higher priority than the non-real time one. This example of self-configuration shows that through cross-layer support, a self-organising networking system can enhance its overall performances which is not possible through strict-layer approaches.

Self-healing Self-healing is the mechanism that allows to detect, localise, and repair failures automatically, primarily distinguished by the cause of the failure. For a network, self-organisation essentially means being able to react to such failure situations automatically which means they needs to be self-healed and MANETs shows that property. From a MANET perspective, self-organisation is essential to enforce routes that need to be persistent in time: self-organisation makes the MANET self-healing with respect to link breakdowns caused by nodes mobility or power failure of intermediate route nodes.

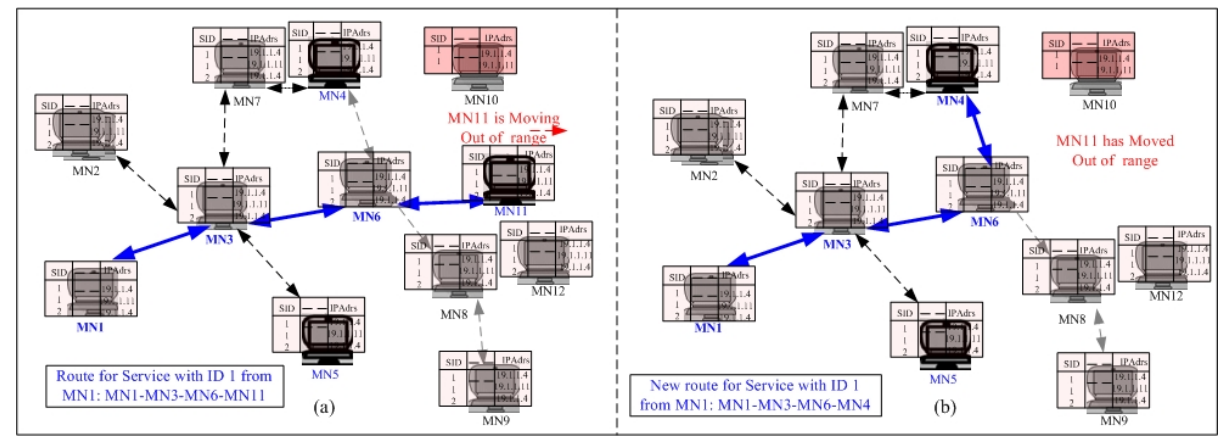

Fig. 5. Sample network scenario one considered for self-healing

In the following we use CLSR to show the enhancement in self-organisation/selfhealing for MANETs. For this case we are considering the scenario as shown in figure 5(a). It consists of twelve nodes and three of them (black coloured nodes), provide three different types of services and others are client nodes. In particular node MN4 and MN11 provide x-type of service which has a service ID (SID) 1. This figure also shows the service table which is available at the network layer of each node and this is contributed by the cross-layer service discoveries and global view formation which were initiated by the node MN1 and MN9 at different times for the service requests for services with service ID 1 and 2 respectively. Finally, MN1 follows the route MN1-MN3-MN6-MN11 to get the service from the node MN11. During this service, sometime around 150s MN11 moves out of range of the network and with the help of CLSR routing scheme and global information all the nodes re-organise themselves similar to figure 5(b). After this organisation, even at the absence of the MN11 node; CLSR and AODV can still support x-type service through MN4 as it can provide the same service. As CLSR exploits proactive route maintenance and service level information at the network layer, so it will the service with lesser control overhead than AODV. As all the nodes (after the move out of MN11) are getting x-type service from 
MN4, so the chances of congestion increases to the links towards MN4 and thus throughput decreases little bit for all the approaches as shown in figure 7 .

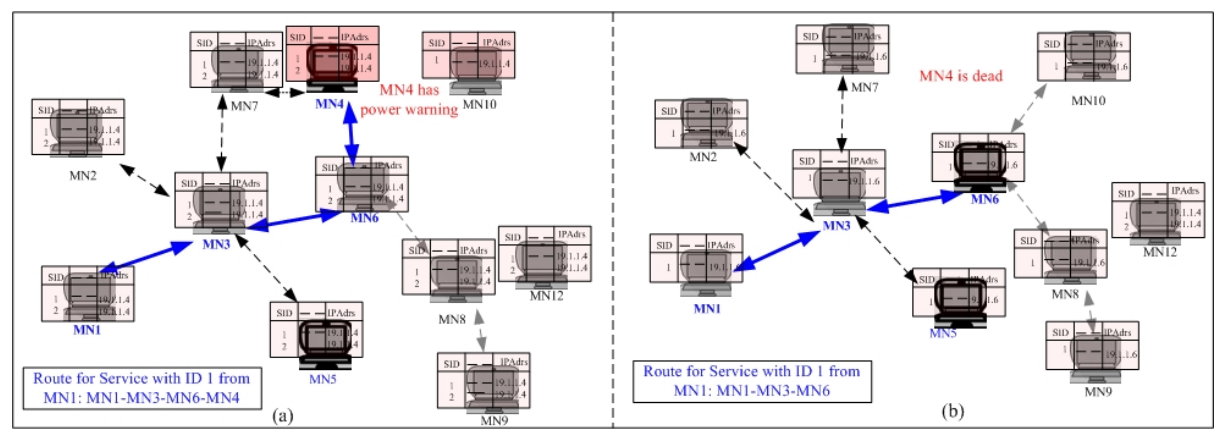

Fig. 6. Sample network scenario two considered for self-healing

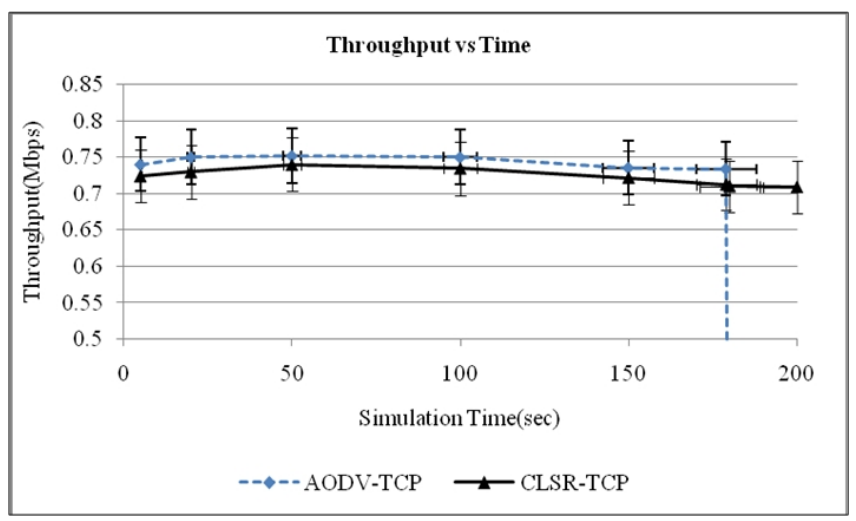

Fig. 7. Throughput for sample network scenario

After the move out of MN11, MN4 over hit by the service requesters and it loses power and finally dies at around 180s. At this situation AODV would not be able to provide $\mathrm{x}$-type service to any node of the network due to lack of any service providing node. On the other hand in CLSR, before the complete dead of the node MN4 predicts its probable power failure and checks service level information to know whether any server for this service is available or not. If there is no node to provide $\mathrm{x}$-type service then MN4 initiates the CLSR's service replications to node MN6 (here we are assuming that all nodes are able to receive service/data replications). After this service replication, service information table will be changed at MN6 and this change initiates the gossiping (the dissemination approach in cross-layer architecture) and with the help of disseminated service level information most of the nodes update their service table as 
shown in figure 6. Finally, MN1 gets the x-type service from MN6. This whole process is can be easily defined as the self-healing in the concerned network and this came with the help enhanced self-organisation within the nodes through cross-layering. As shown in figure 7, there is no service for MN1 after 180s when we use AODV but for CLSR throughput remains almost similar for all the time. This is enhancement in the performance comes through cross-layering at the cost of extra overhead and this due to service replication.

\section{Conclusions}

MANET architectures are increasingly popular in many area of communications, but pose significant challenges in terms of management. They thus provide a perfect environment within which to deploy and experiment with "self-*" properties. While most of the existing works have focused on providing self-organisation using layered design, we believe that cross-layer designs can offer improvements by taking account of the context information collected from multiple different sources. We have implemented a cross-layer routing protocol and compared it with a well-studied layered protocol. To demonstrate the potentials of the crosslayer design approach in enhancing the self-organising properties of wireless networking such as MANETs, we did simulations to show three different aspects (route, self-configuration and self-healing) of a self-organising networking system. Results show the possible improvements in their respective aspects which ultimately enhance the self-organising properties in MANETs like wireless networking environments. These improvements are contributed by the advantages of cross-layer protocol, most notably the prediction and proactive masking of faults caused by node movement and power loss. By adapting routing and service bindings in CLSR, we have been able to demonstrate that cross-layered design can synthesise information in order to improve network behaviour in a way that is difficult to integrate into layered approaches.

What our work does not provide is a design methodology for replacing the information hiding and modularity inherent in layered designs. It certainly seems possible that cross-layer designs will suffer from "spaghetti code" design flaws and a consequent reduction in their maintainability, and this is a subject we wish to explore further in future. We also need to understand the way in which different adaptations are triggered by environmental changes, so that we can predict the adaptive network's performance and robustness more accurately.

\section{Acknowledgments}

This work is partially supported by Science Foundation Ireland under grant number 04/RPI/1544, "Secure and Predictable Pervasive Computing" and Higher Education Authority PRTLI4 under grant number R10891, "NEMBES: Networked Embedded Systems." 


\section{References}

1. The network simulator. http://www.isi.edu/nsnam/ns/ns-build.html.

2. $\operatorname{Rfc}(3561)$ for AODV. http://rfc.dotsrc.org/rfc/rfc3561.html.

3. What is bandwidth and how does it affect VOIP? http://www.quickstartvoip.com/articles/what-is-bandwidth.html.

4. Mehran Abolhasan, Tadeusz Wysocki, and Eryk Dutkiewicz. A review of routing protocols for mobile ad hoc networks. Ad Hoc Networks, 2:1-22, 2004.

5. Josh Broch, David A. Maltz, David B. Johnson, Yih-Chun Hu, and Jorjeta Jetcheva. A performance comparison of multi-hop wireless ad hoc network protocols. In Mobile Computing and Networking, pages 85-97, 1998.

6. W. Chen C. Sadler and L. Kant. Cross-layer self-healing in a wireless ad-hoc network. In Proceedings of World Wireless Congress, 2005.

7. Z J Cai, M Lu, and X D Wang. Channel access-based self-organized clustering in ad hoc network. IEEE Transaction on Mobile Computing, 2:102-113, 2003.

8. K. Chen, S. Shah, and K. Nahrstedt. Cross-layer design for data accessibility in mobile ad hoc networks. In Proceedings of 5th World multiconference on systemics, cybernetics and informatics, 2001.

9. S.E. Czerwinski, B.Y. Zhao, T.D. Hodes, A.D. Joseph, and R.H. Katz. An architecture for a secure service discovery service. In Proceedings of MobiCom, 1999.

10. Falko Dressler. Self-organization in ad hoc networks: Overview and classification. Technical report, University of Erlangen, Martensstr. Germany, 2006.

11. J. Antonio Garcia-Macias and Dante Arias Torres. Service discovery in mobile ad hoc networks: Better at the network layer? In ICPPW '05: Proceedings of the 2005 International Conference on Parallel Processing Workshops (ICPPW'05), pages 452-457, Washington, DC, USA, 2005. IEEE Computer Society.

12. Liljana Gavrilovska. Cross-layering approaches in wireless ad hoc networks. Wireless Personal Communications, 37(3-4):271-290, May 2006.

13. X. Gu, X. Fu, H. Tshofenig, and L. Wolf. Towards self-optimizing protocol stack for autonomic communication: Initial experience. In Proceedings of the 2nd IFIP International Workshop on Autonomic Communication, volume 3854 of LNCS, pages 183-201. Springer-Verlag, October 2005.

14. E. Guttman. Service location protocol: automatic discovery of IP network services. IEEE Internet Computing, pages 71-80, 1999.

15. Zygmunt J. Haas. A new routing protocol for the reconfigurable wireless networks. In Proceedings of 6th IEEE International Conference on Universal Personal Communications, pages 562-566, 1997.

16. Gertjan P. Halkes, Aline Baggio, and Koen Langendoen. A simulation study of integrated service discovery. In EuroSSC, pages 39-53, 2006.

17. F. Heylighen and C. Gershenson. The meaning of self-organization in computing. citeseer.ist.psu.edu/heylighen03meaning.html.

18. Francis Heylighen. The science of self-organization and adaptivity. In Knowledge Management, Organizational Intelligence and Learning, and Complexity, in: The Encyclopedia of Life Support Systems, EOLSS, pages 253-280. Publishers Co. Ltd, 2003.

19. J.L. Van Den Berg, O. Linnell, M. Amirijoo, H. van den Berg, R. Litjens, A. Eisenblatter, C. Blondia, T. Kurner, J. Oszmianski, and C. Schwettz. Self-Organisation in Future Mobile Communication Networks . In ICT - Mobile Summit, 2008.

20. Manish Kochhal, Loren Schwiebert, and Sandeep Gupta. Role-based hierarchical self organization for wireless ad hoc sensor networks. In Proceedings of the 2nd 
ACM international conference on Wireless sensor networks and applications, pages 98-107. ACM Press, 2003.

21. P. Mani and D.W Petr. Development and performance characterization of enhanced AODV routing for CBR and TCP traffic. In Proceedings of the 2004 IEEE Wireless Telecommunications Symposium, 2004.

22. A. Bruce Mcdonald and Taieb Znati. A mobility based framework for adaptive clustering in wireless ad-hoc networks. IEEE Journal on Selected Areas in Communications, 17:1466-1487, 1999.

23. P. Mehra, A. Zakhor, and C. Vleeschouwer. Receiver-driven bandwidth sharing for tcp. In In IEEE INFOCOM, 2003.

24. C. Prehofer and C. Bettstetter. Self-organization in communication networks: principles and design paradigms. Communications Magazine, IEEE, 43(7):78-85, 2005.

25. K Premaratne, J S Zhang, and M Dogruel. Location information-aided taskoriented self-organization of ad hoc sensor systems. IEEE Sensors Journal, 4:85-95, 2004.

26. M.A. Razzaque, Simon Dobson, and Paddy Nixon. A cross-layer architecture for autonomic communications. In Autonomic Networking, volume 4195 of LNCS, pages 25-35. Springer-Verlag, 2006.

27. M.A. Razzaque, Simon Dobson, and Paddy Nixon. Cross-layer optimisations for autonomic networks. Autonomic Communications. Monique Calisti, John Strassner and Sven van der Meer (ed). Birkhauser, 2008.

28. M.A. Razzaque, Simon Dobson, and Paddy Nixon. Cross-layer self routing: a selfmanaged routing approach for MANETs. In 4th IEEE International Conference on Wireless and Mobile Computing, Networking and Communications, 2008.

29. Zhi Ren, Jing Su, and Wei Gu. A cross-layer AODV routing protocol. In Mechatronics and Automation, IEEE International Conference, volume 4, August 2005.

30. Mohamed Abou El Saoud, Thomas Kunz, and Samy Mahmoud. SLPManet: service location protocol for MANET. In Proceedings of the 2006 international conference on Wireless communications and mobile computing, pages 701-706, 2006.

31. S. Schmid, M. Sifalakis, and D. Hutchison. Towards Autonomic Networks. In Autonomic Networking, volume 4195 of LNCS, pages 1-11. Springer-Verlag, 2006.

32. V. Srivastava and Motani. Cross-layer design: a survey and the road ahead. Communications Magazine, IEEE, 43(12):112-119, 2005.

33. Hong Tang and Huaglory Tianfield. Self-organizing networks of communications and computing. ITSSA, 1(4):421-431, 2006.

34. Rainer Unland and Huaglory Tianfield. Shaping the future: Towards the next generation of powerful, pervasive, and service-oriented distributed systems. Multiagent Grid Syst., 1(3):225-229, 2005.

35. Alex Varshavsky, Bradley Reid, and Eyal de Lara. A Cross-Layer Approach to Service Discovery and Selection in MANETs. In The Second International Conference on Mobile Ad-Hoc and Sensor Systems, November 2005. 